\title{
Comparative analysis of mast cell count in normal oral mucosa and oral pyogenic granuloma
}

\author{
Reet Kamal ${ }^{1}$, Parveen Dahiya ${ }^{2}$, Sangeeta Palaskar ${ }^{2}$, V.P. Shetty ${ }^{2}$ \\ ${ }^{1}$ Senior Lecturer. Dept. of Oral Pathology, HIDS, Paonta Sahib. \\ ${ }^{2}$ Doctor. Dept. of Oral \& maxillofacial Pathology, HIDS, Paonta Sahib.
}

\author{
Correspondence: \\ Deptt. of Oral \& maxillofacial pathology, \\ HIDS, Paonta Sahib. Distt.-Sirmour.(Himachal Pradesh) \\ INDIA \\ Email: parveen_132@yahoo.com
}

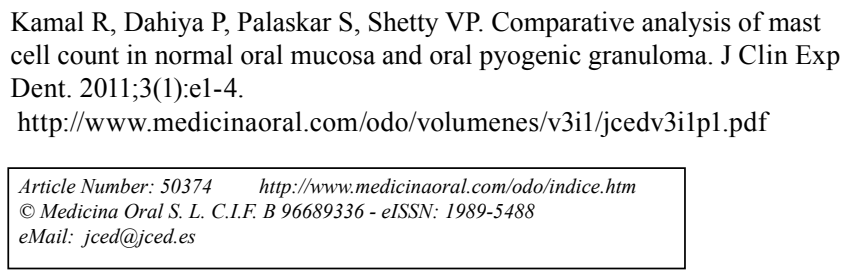

\begin{abstract}
Introduction: Mast cells are large granular cells that arise from a multipotent CD 34+ precursor in the bone marrow normally distributed throughout connective tissues. The most common method to study role of mast cells in any altered condition involves their identification and quantification in that condition and compare the values with that of the normal average count or number of mast cells. The present study was thus, undertaken to identify as well as quantify mast cells in oral pyogenic granuloma and compare it with the average count of mast cells in normal oral mucosa, thus aiming to assess the changes in count of mast cells in oral pyogenic granuloma.

Materials and Methods: Ten cases of normal oral mucosa and thirty cases of oral pyogenic granuloma were studied for mast cell number using $1 \%$ toluidine blue.

Results: An increase in mast cell number was observed in oral pyogenic granuloma. The mast cell count/high power field in pyogenic granuloma and normal oral mucosa was 10.27 and 4.58 respectively. There is a statistically significant increase in the mean of average mast cell count per high power field in oral pyogenic granuloma in comparison to normal oral mucosa. These facts may morphologically indicate a possibility of a role of mast cells in angiogenesis and recruitment of inflammatory cells which are characteristic features of oral pyogenic granuloma
\end{abstract}

Key words: Mast cells, oral pyogenic granuloma, degranulated, inflammation. 


\section{Introduction}

Mast cells are large granular cells that arise from a multipotent $\mathrm{CD} 34+$ precursor in the bone marrow and are normally distributed throughout connective tissues(1). Mast cell count or densities have been studied in the connective tissues of cutaneous pyogenic granuloma. The results of these studies indicated that average mast cell numbers or densities were altered in the connective tissues of cutaneous pyogenic granuloma in comparison to the normal average count of mast cells. Based on these results, it was proposed that mast cells may play a role in pathogenesis of cutaneous pyogenic granuloma by recruiting inflammatory cells and promoting neoangiogenesis (2-3). The role of mast cells, however, in oral reactive conditions like pyogenic granuloma is still obscure. The present study was thus, undertaken to identify as well as quantify mast cells in oral pyogenic granuloma and compare it with the average count of mast cells in normal oral mucosa, thus aiming to assess the role of mast cells in pathogenesis of pyogenic granuloma.

The study aimed at determining the average mast cell count in normal oral mucosa and oral pyogenic granuloma. The objectives of the study were to determine the average mast cell count in normal oral mucosa, to determine the average mast cell count in oral pyogenic granuloma and to determine the average count of intact and degranulated mast cells in in oral pyogenic granuloma.

\section{Materials and Methods}

The present study consisted of 30 previously diagnosed paraffin blocks of oral pyogenic granuloma obtained from the records of M.M.C. D.S.R., Mullana and $10 \mathrm{ca}-$ ses of normal oral mucosa (gingiva).

Slides of normal oral mucosa and pyogenic granuloma cases were subjected to microscopic evaluation- one being hematoxylin and eosin stained slide and the other being toluidine blue stained slide of each case. The slides were observed under binocular research microscope under different magnifications.

Toluidine blue stained sections: Two morphological types of mast cells could be recognized in toluidine blue stained sections- intact dark blue/purple cells which do not show any signs of degranulation and degranulating mast cells with more extruded metachromatic granules visible adjacent to cell. The latter type shows partial or complete disintegration of original cell outline. Toluidine blue stained sections of pyogenic granuloma and normal oral mucosa were analyzed under different magnifications and five fields were selected from the entire connective tissue.

Mast cells counting: Both intact as well as degranulated mast cells as well as the total number of mast cells in the 5 high power fields of each section of normal oral mucosa (Fig. 1) and oral pyogenic granuloma (Fig. 2) were recorded under $40 \mathrm{X}$ magnification. Particular attention was put not to overlap mast cell count. Results were expressed as the average number of mast cells per high power field.

Statistical analysis: The means of the average mast cell count per high power field in normal oral mucosa and pyogenic granuloma were compared by using ' $t$ ' test. Means of the average mast cell count of intact and degranulated mast cells per high power field in normal oral mucosa and pyogenic granuloma were also compared by using ' $\mathrm{t}$ ' test.

\section{Results}

Out of 30 cases of oral pyogenic granuloma, high degree of occurrence was observed in the 3rd, 4th and 5th decade with more than $70 \%$ of the cases affecting this age group with a female predilection of 2:1. Most of the cases ( $>80 \%)$ occurred on gingiva. Duration of 30 cases of oral pyogenic granuloma ranged from 10 days to two years.

In normal oral mucosa, the average number of mast cells per high power field was 4.58 and 10.27 in pyogenic

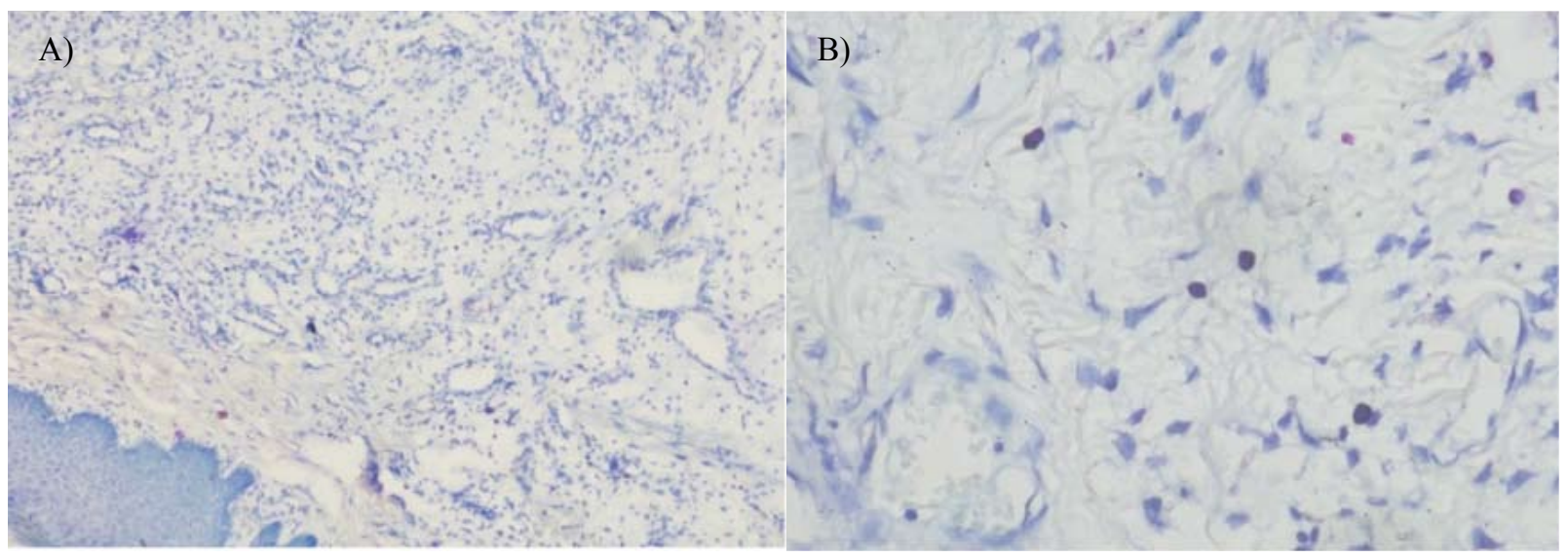

Fig. 1. Photomicrographs of mast cells in normal oral mucosa

a. Mast cells in normal oral mucosa (10X)

b. Mast cells in normal oral mucosa (40X) 


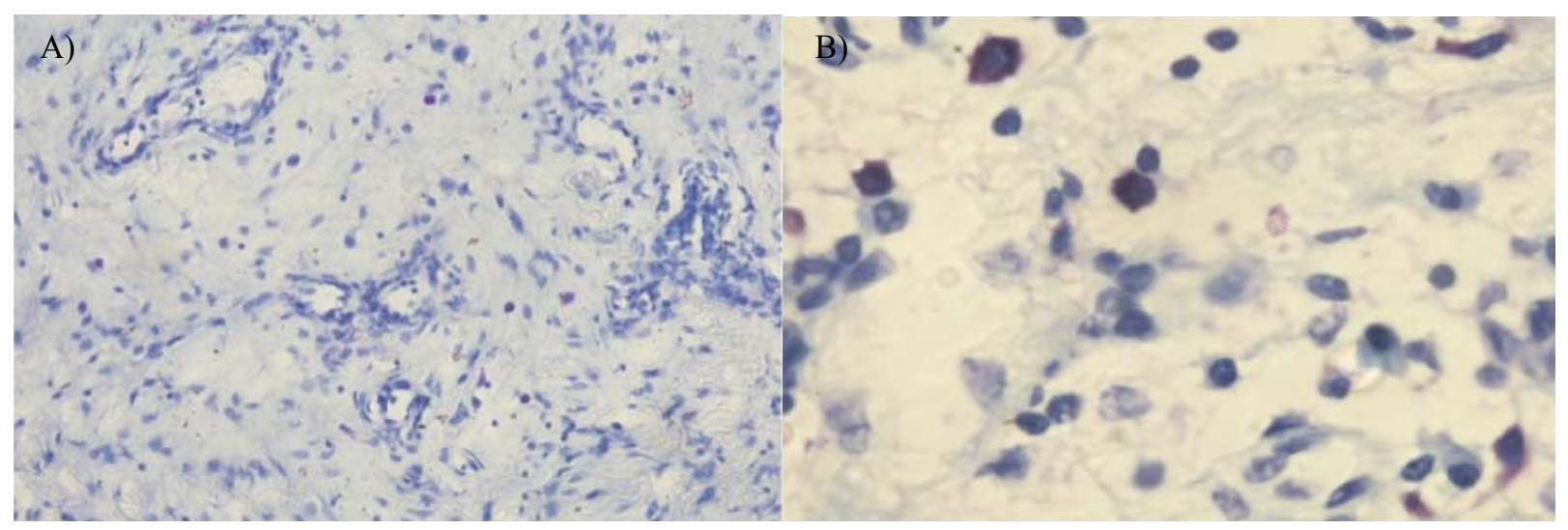

Fig. 2. Photomicrographs of mast cells in oral pyogenic granuloma a. $20 X$

b. $100 X$

granuloma. The minimum number of mast cells per high power field was 4.20 and 1.80 in pyogenic granuloma and normal oral mucosa and the maximum number of mast cells per high power field was 6.20 and 17.20 in pyogenic granuloma and normal oral mucosa respectively. The ' $t$ ' value was calculated while comparing mean mast cell count in pyogenic granuloma and normal oral mucosa. The 't' value of -5.348 was found to be highly significant.

In normal oral mucosa, the average number of intact mast cells per high power field was 2.72 in normal oral mucosa and 4.25 in pyogenic granuloma. The ' $t$ ' value was calculated to compare mean intact mast cell count in pyogenic granuloma and normal oral mucosa. ' $t$ ' value of -1.675 was found to be significant. In normal oral mucosa, the average number of degranulated mast cells per high power field was 1.86 in normal oral mucosa and 6.27 in pyogenic granuloma. The ' $t$ ' value of -5.040 while comparing mean mast cell count in pyogenic granuloma and normal oral mucosa was found to be highly significant.

\section{Discussion}

Pathogenesis of pyogenic granuloma is still debatable. Whether it represents a benign neoplasm, an infectious process or a reactive lesion remains unclear.

An increase in average mast cell count has been observed in inflammatory reactive conditions like inflammatory hyperplasia, granulation tissue, gingivitis (4) as well as in vascular conditions like hemangiomas (5) suggesting that mast cells may play a role in recruitment of inflammatory cells and angiogenesis.

Role of mast cells have been studied in pathogenesis of cutaneous pyogenic granuloma by calculating the average count of mast cells but role of mast cells in pathogenesis of oral pyogenic granuloma has not been studied in a similar manner. Hence, this study was undertaken to quantify mast cells and assess their role in pathogenesis of pyogenic granuloma.

Also, as stated by Krishnaswamy et al. (1) degranulation of mast cells releases preformed granules containing mediators such as histamine, tumor necrosis factor, serotonin and numerous proteases responsible for most of the mast cell dependent functional responses. Therefore, determining whether mast cell is intact or degranulated may be a good indicator to assess whether mast cells are involved in a particular biological process. Thus, in this study, toluidine blue stain was selected to demonstrate intact mast cells and their granules as well as degranulation of mast cells.

Biviji (6) in his study on leukoplakia observed an increase in average mast cell count and suggested that stimulated mast cells may release IL-1, which causes increased epithelial proliferation. Caffesse et al. (7) observed in their study that the presence of inflammation was associated with an increase in epithelial mitotic activity in the gingiva. Roberts and Brenchley (8) in their study observed an increase in the average mast cell count in renal fibrosis and stated that mast cell release fibrogenic factors and induce fibroblast proliferation. Glowacki and Mulliken (9) in their study on average count of mast cells in hemangiomas and vascular proliferations observed that hemangiomas are characterized by an increase in the average number of mast cell count. They suggested that release of mediators from mast cells may lead to neoangiogenesis. de Oliveira Rodini et al.(10) in their study found an increase in the average mast cell count in the periapical inflammatory lesions. They proposed that mast cells may lead to inflammatory changes by release of their chemical mediators.

In the present study, the ' $t$ ' value for comparison of the means of average number of mast cells per high power field in normal oral mucosa and pyogenic granuloma was found to be highly significant. The results of the present study were comparable to Shea et al. (3), Ribatti et al. (11) and Hagiwara et al. (5) who reported an in- 
crease in the number of mast cells in pyogenic granuloma. But the results are in sharp contrast to Patrice et al. (2) who reported normal number of mast cells. The discordant results regarding mast cell density in these studies may be attributed to several reasons: A difference in criteria regarding borderline positive cells between observers. Some have counted only mast cells having both the nucleus and metachromatic cytoplasmic granules present in the same section and disregarded clusters of metachromatic granules without a visible nucleus, a difference in the staining method or a difference in vascularity of the area examined.

Calculation of ' $t$ ' values for comparing of means of average count of intact and degranulated mast cells per high power field in normal mucosa and pyogenic granuloma revealed that ' $t$ ' value was more significant in case of degranulated cells than in case of intact mast cells. This suggests that mean count of degranulated mast cells were more significantly raised than that of intact mast cells in pyogenic granuloma. These results may imply that it is only when mast cells are activated or degranulated, lead to inflammatory and vascular changes in the connective tissue.

This data, thus suggest that mast cells on stimulation by various etiological factors may increase in number and subsequently undergo degranulation and cause inflammatory and vascular changes leading to formation of pyogenic granuloma, the mechanism of which may be proposed as follows.

Mast cells are responsive to neuropeptides and through their interaction with neural elements, form a neural immune network with Langerhans cells in mucosal tissues. This facilitates mast cell degranulation in response to a range of immunological and non-immunological stimuli as a result of various etiological factors. Further, on degranulation, mast cells release via their granules a range of pre-formed mediators, including cytokines, vasoactive amines and enzymes (12). These mediators lead to inflammatory and vascular changes in pyogenic granuloma.

Also, on the basis of clinical observations, etiopathogenesis of pyogenic granuloma appears less likely to be of infectious origin as suppuration was not associated with any of the cases. On the basis of histopathological observations, theory of neoplastic origin also appears questionable as it was observed that long standing cases were showing more connective tissue fibrosis compared to cases of short duration. On the basis of mast cell related observations, it appears that pyogenic granuloma represents a reactive lesion resulting from local etiological factors like gingival inflammation, calculus or trauma which activate mast cells resulting in release of mast cell mediators which further leads to subsequent changes in the tissues leading to formation of pyogenic granuloma. Further investigations into mast cell derived angiogenic factors and mast cell mediators responsible for recruiting inflammatory cells, might provide a better understanding of the role of mast cells in inflammatory vascular lesions like pyogenic granuloma.

The significant increase in the average mast cell count per microscopic field in pyogenic granuloma in comparison to normal oral mucosa strengthens the possibility of a role of mast cells in the pathogenesis of pyogenic granuloma. The significant ' $t$ ' values of degranulated mast cells per microscopic field may imply that degranulation of mast cells may be responsible for recruitment of inflammatory cells and angiogenesis which are characteristic features seen in the connective tissue of pyogenic granuloma.

However, further exploration is required to know the exact role of mast cells in inflammation, in vascularity and thus, in turn, in pyogenic granulomas of oral cavity.

\section{References}

1. Krishnaswamy G, Kelley J, Johnson D, Youngberg G, Stone W, Huang SK, et al. The human mast cell: functions in physiology and disease. Front Biosci. 2001;6:D1109-27.

2. Patrice SJ, Wiss K, Mulliken JB. Pyogenic granuloma (lobular capillary hemangioma): a clinicopathologic study of 178 cases. Pediatr Dermatol. 1991;8:267-76.

3. Shea CR, Prieto VG. Mast cells in angiolipomas and hemangiomas of human skin: are they important for angiogenesis? J Cutan Pathol. 1994;21:247-51.

4. Klatt EC, Lukes RJ, Meyer PR. Benign and malignant mast cell proliferations. Diagnosis and separation using a $\mathrm{pH}$-dependent toluidine blue stain in tissue section. Cancer. 1983;51:1119-24.

5. Hagiwara K, Khaskhely NM, Uezato H, Nonaka S. Mast cell "densities" in vascular proliferations: a preliminary study of pyogenic granuloma, portwine stain, cavernous hemangioma, cherry angioma, Kaposi's sarcoma, and malignant hemangioendothelioma. J Dermatol. 1999;26:577-86.

6. Biviji AT. .Mast cells in normal and leukoplakic buccal mucosa. J Indian Dent Assoc. 1973;45:189-91.

7. Caffesse RG, Nasjleti CE, Kowalski CJ. Carrageenan-induced inflammation and its effects on mitotic activity and keratinization of gingival epithelium. A histologic and autoradiographic study. J Periodontol. 1985;56:132-8

8. Roberts IS, Brenchley PE.Mast cells: the forgotten cells of renal fibrosis. J Clin Pathol. 2000;53:858-62.

9. Glowacki J, Mulliken JB. Mast cells in hemangiomas and vascular malformations. Pediatrics. 1982;70:48-51.

10. de Oliveira Rodini C, Batista AC, Lara VS. Comparative immunohistochemical study of the presence of mast cells in apical granulomas and periapical cysts: Possible role of mast cells in course of human periapical lesions. Oral Surg Oral Med Oral Pathol Oral Radiol Endod 2004;97: 59-63.

11. Ribatti D, Vacca A, Schiraldi G, Sorino S, Caprio F, Mazzotta F, et al. Pyogenic granuloma stimulates angiogenesis in the chick embryo chorioallantoic membrane. Int J Microcirc Clin Exp. 1996;16:82-8

12. Walsh LJ, Davis MF, Xu LJ, Savage NW. Relationship between mast cell degranulation and inflammation in the oral cavity. $\mathrm{J}$ Oral Pathol Med 1995;24:266-272. 